\title{
DISPERSIÓN DE LUZ POR UNA NANOPARTÍCULA DE PLATA CON FORMA ESFEROIDAL PROLATA
}

\author{
Light scattering by a silver nanoparticle prolate spheroidal shape
}

EPISTEMUS

ISSN: 2007-8196 (electrónico)

ISSN: 2007-4530 (impresa)

Alejandro Castellanos Jaramillo ${ }^{1}$

Arnulfo Castellanos Moreno ${ }^{2}$

Recibido: 15 de marzo de 2016,

Aceptado: 30 de mayo de 2016

\section{Autor de Correspondencia:}

Dr. Arnulfo Castellanos Moreno

Correo: acastell@correo.fisica.uson.mx

\section{Resumen}

Usando el método de diferencias finitas en el dominio del tiempo se estudia la incidencia y dispersión de radiación electromagnética de una nanopartícula de plata con forma esferoidal prolata. Se obtiene la distribución de frecuencias del vector de Poynting dispersado en el régimen casi estacionario y se estudia el estado de radiación de la nanopartícula cuando el pulso de luz ha dejado de perturbarla. Los resultados son de interés didáctico.

Palabras clave: dispersión de luz, nanoóptica, nanopartículas, plasmones de superficie.

\section{Abstract}

Using the Finite Difference in the Time Domain method it is possible to study the incidence and dispersion of electromagnetic radiation of a nanoparticle of spheroidal shape. One can obtain the frequency distribution of the Poynting vector dispersed in the quasi-stationary regime, and can study the radiation state of the particle when the light pulse has stopped perturbing it. The results are of didactic interest.

Keywords: light scattering; nano-optics; nanoparticles; surface plasmones. 


\section{INTRODUCCIÓN}

En este trabajo abordamos un sistema físico que consiste en un pulso de luz que incide sobre una nanopartícula de plata de forma esferoidal prolata, la excita y la deja radiando energía. Reportamos los resultados de la solución numérica de las ecuaciones de Maxwell por medio del método de diferencias finitas en el dominio del tiempo (FDTD por sus siglas en inglés). Mostramos cómo absorbe energía y dónde se coloca su máximo de absorción para sendos valores específicos de su eje mayor y menor. Enseguida se hace un análisis de la actividad electromagnética en el entorno cercano y dentro de la nanopartícula. Los resultados son de utilidad didáctica.

En esencia, el problema que abordamos es un fenómeno de dispersión de ondas electromagnéticas por parte de una nanopartícula. La presencia de las nanoestructuras está presente en la civilización humana al menos desde la época de los romanos, por ejemplo, en las copas de vidrio conocido como lycurgus, que presentan tonalidades de colores que provienen de la presencia de nanopartículas de plata que dispersan la luz [1], dando las tonalidades bellas que pueden ser admiradas en el Museo Británico (British Museum).

La dispersión de luz por nanopartículas genera una gama muy amplia de expectativas en la tecnología actual [2]. Entre sus aplicaciones se mencionan desde terapias novedosas en la física médica, hasta usos diversos en la optoelectrónica, incluyendo nanoantenas y puertas lógicas, de modo tal que se espera el diseño y próxima producción de dispositivos que utilizarían ondas electromagnéticas para transportar señales dentro de chips en lugar de mover electrones, los cuales presentan la bien conocida disipación de energía. Recientemente ha sido posible transferir energía a partir de alambres cuánticos hacia un tipo de enzimas oxidativas que producen luminiscencia, siendo este un logro que es considerado sumamente eficiente [3]. También se ha logrado combinar puntos cuánticos con moléculas orgánicas para mejorar la captura de energía solar [4]. En consecuencia con lo anterior, uno de los objetivos más recientes ha sido la producción de nanopartículas de distintos materiales y tamaños que son elaborados con diversas técnicas.

La ciencia necesaria para comprender estos fenómenos también debe ser novedosa, pues las dimensiones de estas nanoestructuras es tal que los efectos de superficie toman relevancia y compiten con los del volumen. Esto se debe a que las nanopartículas metálicas, sobre las cuales incide luz, presentan varias frecuencias características que deben ser consideradas: 1) la frecuencia de la onda electromagnética, $n ; 2)$ la frecuencia de colisión de los electrones con los fonones y de los electrones entre si, $\left(n_{e f} y n_{e e}\right)$, 3) la frecuencia de vibración media, $n_{5}$, debido al choque de los electrones con la superficie de la nanopartícula. Dicha frecuencia $n_{s}$ toma importancia cuando la trayectoria libre media de los electrones de conducción del metal es mayor que las dimensiones de la nanoestructura [5]. En este caso, la forma de la nanopartícula resulta importante, dando lugar a que sea necesario revisar los conceptos fundamentales de la física estadística, que usualmente son desarrollados sobre la base de que las propiedades termodinámicas son independientes de la forma del sistema físico bajo estudio [6].

La nueva literatura ha llevado a que revistas dirigidas a profesores y a estudiantes no graduados traten de listar fuentes de información, a elaborar glosarios y diseñar videos con los que buscan facilitar la comprensión de estas temáticas por parte de las generaciones en proceso de formación científica [7]-[9]. Más aún, hay autores que han intentado exponer la historia del posible camino conceptual que lleva hacia la nanotecnología [10]. Así se ha generado un lenguaje nuevo también, pero su uso cotidiano no implica que es bien entendido por los estudiantes. Por lo tanto, otro de los objetivos de este trabajo es abordar la discusión de algunos de estos vocablos, prestando atención al concepto de plasmón y haciendo lo posible por contribuir a su aclaración ante los ojos de quienes se acercan por primera vez a la temática de la nanociencia y la nanotecnología.

El orden del artículo es el siguiente: en la segunda sección presentamos la teoría de diferencias finitas en el dominio del tiempo, el cubo donde se realiza el cálculo numérico, la forma de la celda de Yee y la del pulso electromagnético que se utiliza. En la tercera sección se abordan algunos aspectos relevantes: el concepto de plasmón y el modelo de Drude para los electrones de conducción de un metal. En la cuarta sección se presentan los resultados y la discusión de los mismos. Finalmente, se dedica la quinta sección a presentar las conclusiones de este trabajo.

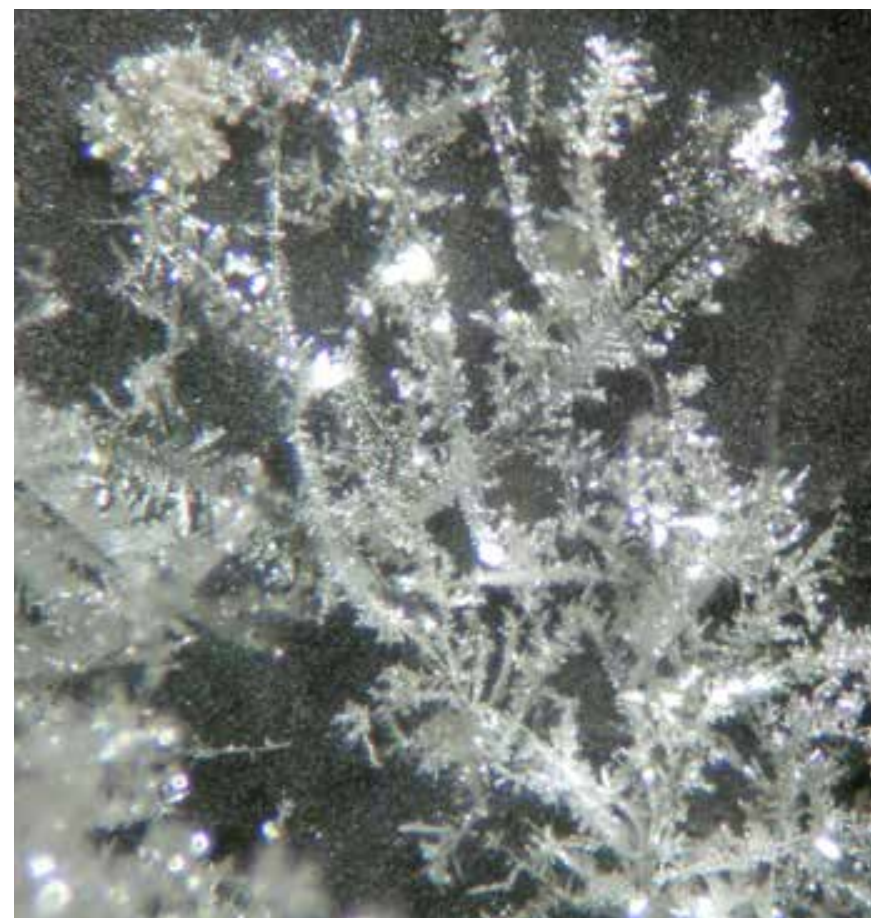




\section{TEORÍA}

Consideramos un cubo de $320 \mathrm{~nm}$ de arista que tiene en su centro una nanopartícula metálica de forma esferoidal prolata. En lo sucesivo éste será llamado: el espacio. Sobre éste se procesa el avance de un pulso electromagnético cuya propagación se calcula resolviendo numéricamente las ecuaciones de Maxwell. En el centro del cubo se encuentra una nanopartícula de forma esferoidal prolata. El pulso surge desde la cara definida por $k=0$, para toda $i, j=, 2, \ldots, 320$ y avanza hacia $k$ creciente.

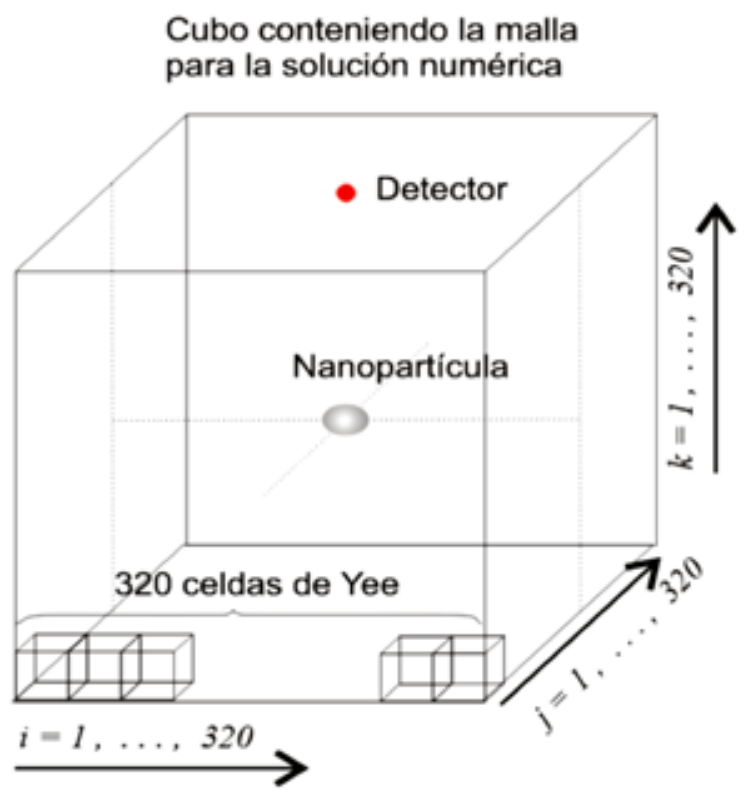

Figura 1. El espacio para la solución numérica es un cubo de arista $320 \mathrm{~nm}$.

Se utiliza la técnica de diferencias finitas en el dominio del tiempo (FDTD por sus siglas en inglés) para obtener la solución aproximada de las ecuaciones de Maxwell. La celda de Yee es el instrumento geométrico fundamental para esta clase de soluciones numéricas [11]. En nuestro caso se trata de un cubo de $1 \mathrm{~nm}$ de arista, de modo que se necesitan $3.2768 \times 10^{7}$ de ellas para realizar el cálculo.

Las ecuaciones de Maxwell que se discretizan son la ley de Faraday y la de Ampere Maxwell [12]:

$$
\begin{aligned}
& \nabla \times \vec{E}=-\frac{\partial \vec{B}}{\partial t} \\
& \nabla \times \vec{H}=\vec{J}+\frac{\partial \vec{D}}{\partial t}
\end{aligned}
$$

Son las que contienen la naturaleza dinámica del campo electromagnético. Estas dos ecuaciones son discretizadas en un arreglo de puntos en un cubo que, como hemos mencionado, recibe el nombre de celda de Yee. La representación usual de ésta se muestra en la figura 2.

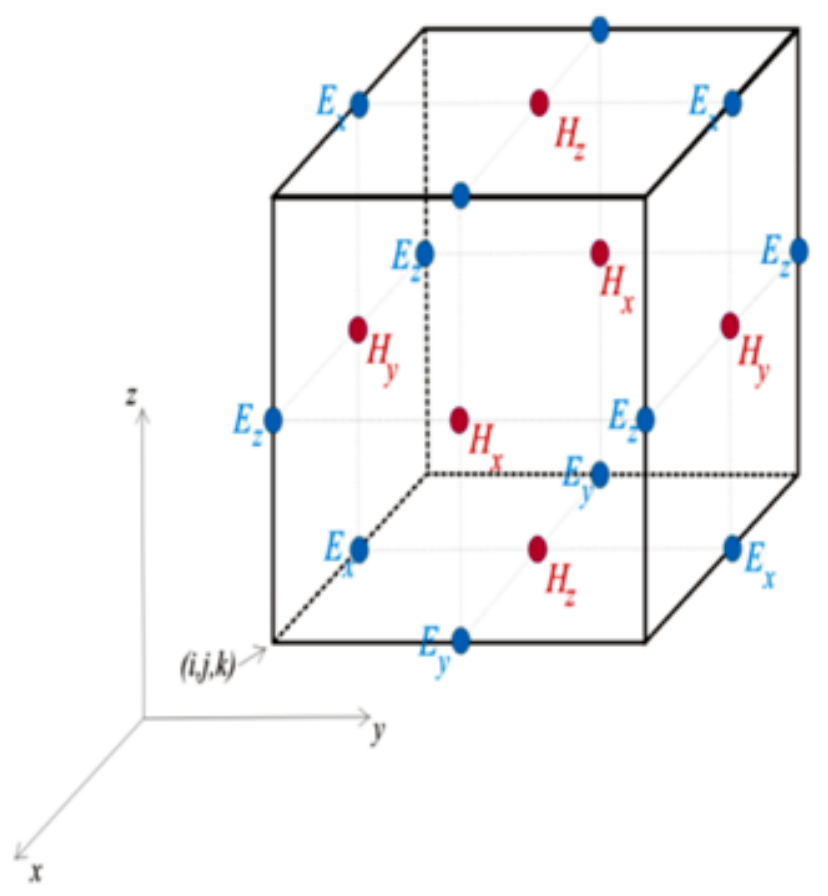

Figura 2. Celda de Yee. Un nanómetro de arista.

Agregamos la discretización de una de las componentes de la ecuación (2) como ejemplo, el resto son similares y pueden ser consultadas en la literatura [13]:

$$
\begin{gathered}
D_{x}^{n}\left(i+\frac{1}{2}, j, k\right)=D_{x}^{n-1}\left(i+\frac{1}{2}, j, k\right) \\
\frac{+\Delta t}{\Delta y}\left[H_{z}^{n-\frac{1}{2}}\left(i+\frac{i}{2}, j+\frac{1}{2}, k\right)-H_{z}^{n-\frac{1}{2}}\left(i+\frac{i}{2}, j-\frac{1}{2}, k\right)\right] \\
-\frac{\Delta t}{\Delta z}\left[H_{y}^{n-\frac{1}{2}}\left(i+\frac{i}{2}, j, k+\frac{1}{2}\right)-H_{y}^{n-\frac{1}{2}}\left(i+\frac{i}{2}, j, k-\frac{1}{2}\right)\right] \\
-\Delta J_{x}^{n-\frac{1}{2}}\left(i+\frac{1}{2}, j, k\right)
\end{gathered}
$$

donde $i$ numera los puntos del espacio en la dirección $x, j$ y $k$ lo hace en la dirección y y en la dirección $z$ respectivamente. Todos los índices corren desde 0 hasta 320. El supra índice $n$ numera la discretización en el tiempo.

La estructura de estas ecuaciones discretas es tal que, en la celda de Yee, las componentes del campo magnético $\{H y, H z\}$ y la densidad de corriente eléctrica, $J_{x}$, ayudan al campo eléctrico $E x$, a actualizarse. Así mismo, el campo eléctrico ayudará al magnético en su proceso de actualización.

El término de la densidad de corriente eléctrica, $J$, contiene la información sobre el material y se actualiza a la vez que los valores de los campos. La base teórica para este trabajo es el modelo de Drude, que explicaremos más adelante. A partir de éste se obtiene la función dieléctrica que presentamos en la ecuación (4), y de esta última, se llega a la ecuación (5), que una vez discretizada, se utiliza para actualizar $J$. 


$$
\begin{aligned}
& \epsilon_{\gamma}=\epsilon_{\infty}-\frac{\omega_{p}^{2}}{\omega^{2}+i \gamma \omega} \\
& \frac{\partial \vec{J}}{\partial t}=-\gamma \vec{J}+\epsilon_{0} \omega_{p}^{2} \vec{E}
\end{aligned}
$$

La discretización de la ecuación (5) es elemental. La forma del pulso que generamos es:

$$
E=E_{0} \operatorname{Sen}^{2}\left(\frac{\pi t}{\tau}\right) \cos (\omega t)
$$

donde $E_{0}=1 \mathrm{Volt} / \mathrm{m}, w=4.7091 \times 10^{1} \square \mathrm{y}$ la duración del pulso es $t=0.36 \times 10^{-15} \mathrm{~s}$. La transformada de Fourier de la expresión (6) es la función que sigue:

$$
G(\omega)=\frac{2 \pi \operatorname{Sen}(\omega t)}{\omega\left(4 \pi^{2}-\omega^{2} \tau^{2}\right)}
$$

y la gráfica de ambas se observa en la figura 3.
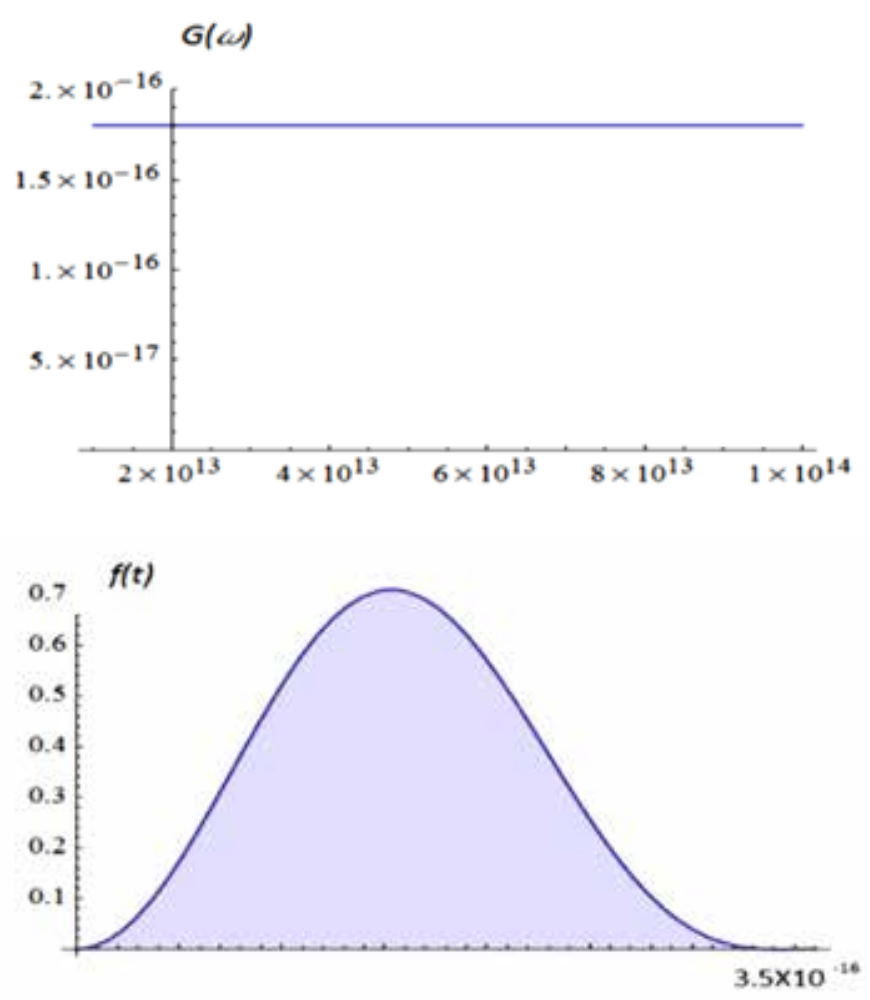

Figura 3. Pulso en el tiempo (izquierda). Transformada de Fourier (derecha).

La virtud de este pulso es que en las frecuencias de interés (radiación infrarroja y visible), tiene una pendiente que es del orden de $10^{-34}$. Es decir, desciende extremadamente lento. Para los fines prácticos de este trabajo, lo anterior da por consecuencia que la naturaleza del pulso sea equivalente al de luz blanca, en el sentido de que todas las frecuencias (colores) tienen casi el mismo peso.

\section{ALGUNOS CONCEPTOS RELEVANTES}

Para simular la propagación de luz dentro de la nanopartícula metálica usaremos el modelo de Drude. Éste se basa en la segunda ley de Newton y su utilidad se debe a que las transiciones de electrones entre dos niveles de un átomo presentan algunas propiedades físicas que son similares a las que resultan del tratamiento matemático de la resonancia de un oscilador armónico amortiguado que está bajo la influencia de un campo electromagnético periódico [12]. Si pensamos únicamente en la más probable de todas las transiciones, estaremos tratando con la más intensa de las líneas de emisión (o absorción) de un sistema atómico que interactúa con un fotón de frecuencia wo. Clásicamente esto se ha formalizado mediante la ecuación de movimiento de un oscilador armónico de frecuencia wo, con una constante de amortiguamiento g y bajo la acción de un campo de la forma Eo cos(wot). La ecuación que describe al sistema es la siguiente:

$$
\frac{d^{2} \vec{x}(t)}{d t^{2}}+\gamma \frac{d \vec{x}(t)}{d t}=\frac{e}{m} \vec{E}(t)
$$

El valor de $g$ se ajusta mediante experimentos y la consecuencia es que se logra describir algunas de las propiedades ópticas de un metal. Cuando el material tiene más de un par de niveles de energía interviniendo en forma relevante para el experimento, se agregan ad hoc otros valores de $g$. La descripción precisa se obtiene mediante la teoría cuántica, como parte del estudio del efecto Stark, que trata de la influencia de campos eléctricos externos sobre sistemas atómicos [14].

En la sección de resultados veremos que la nanopartícula de plata que recibe la onda electromagnética responde formando plasmones en su superficie. Estos son excitaciones colectivas que involucran fluctuaciones en la densidad de carga de un material, de tal modo que sus frecuencias dependen de las propiedades del mismo, y también, de las propiedades geométricas de la nanopartícula, como su tamaño y su forma. Se trata de un acoplamiento con el campo incidente.

Los plasmones son detectados porque la dispersión y la absorción de luz ocurren de manera más intensa en rangos de frecuencia muy específicos. En el caso del trabajo que reportamos. Se calculó el campo dentro y fuera de la nanopartícula y se graficó su intensidad para tiempo algorítmico igual a 103, es decir, justo después de que el pulso enviado abandonó la nanoestructura. Además, se dejó correr el algoritmo de solución numérica hasta que el tiempo alcanzó el valor 105 , enseguida se calculó el vector de Poynting [6] en el punto indicado como: detector, en la figura y se obtuvo un registro temporal de los valores del campo electromagnético. Enseguida se llevó a cabo una transformada de Fourier y se graficó el resultado. Las figuras se muestran más adelante. 


\section{RESULTADOS}

La presentación de resultados de este trabajo resulta más cómoda si se utiliza como unidad de tiempo al lapso que transcurre mientras la luz recorre $0.5 \mathrm{~nm}$. Se llamará tiempo algorítmico (TA) y resulta de dividir $0.5 \times 10^{-9} \mathrm{~m}$ entre la rapidez de la luz en el vacío. El cociente es 1.66782X10-18 s.

Usando cinemática elemental se puede calcular el tiempo que tarda el pulso luminoso en llegar a la pared de la nanopartícula que se encuentra más cerca de la pared emisora. Son 270 unidades de TA. Debido a que el material de la nanopartícula distorsiona y retarda el pulso incidente, no es sencillo calcular con precisión el instante en que el pulso abandona la nanopartícula, pero una estimación cinemática lleva a que eso ocurre cuando han transcurrido aproximadamente 470 unidades de TA. En consecuencia, un archivo con el valor de los campos en el tiempo $t=1000$ unidades de TA, garantiza que estamos registrando las propiedades de una nanopartícula exitada y que el pulso ya no está presente.

Por la razón anterior, el programa de cómputo guardó en archivo los valores del campo electromagnético en 1000 unidades de TA y a partir de esta información fue seleccionado el plano definido por $k=161$, que corta en dos el espacio. Se calculó la magnitud del campo electromagnético y se graficó. El resultado es la figura 5a, que será estudiada más adelante.

La segunda acción fue dejar correr la simulación hasta $10^{5}$ unidades de TA, para guardar en un archivo la magnitud del vector de Poynting en el punto $D$ que se indica en la figura 1. En este caso el proceso es cuasiperiódico y se justifica la realización de un análisis de Fourier. La señal recibida en el tiempo fue sujeta a una transformada de Fourier y se encontró que una nanopartícula de plata de las dimensiones dadas presenta un pico de dispersión que se muestra en la figura 4.

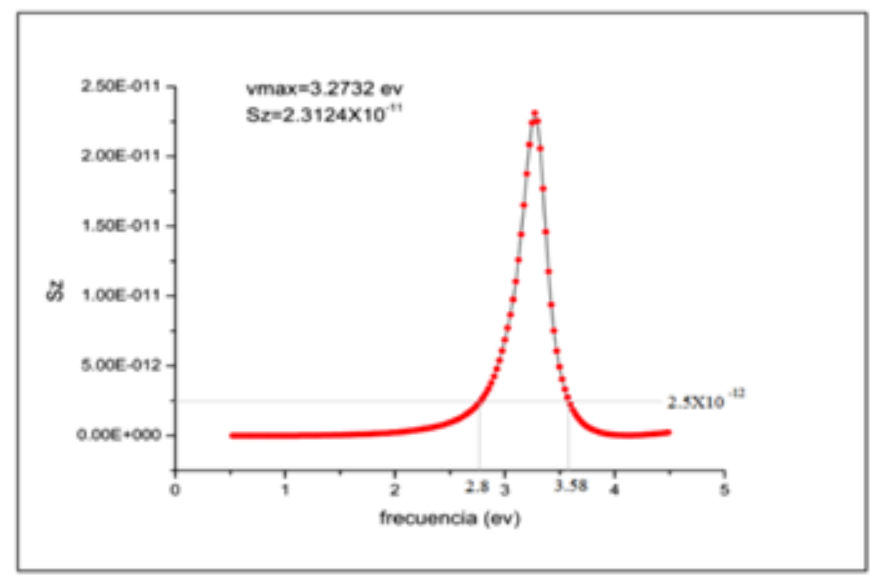

Figura 4. Contribuciones de las frecuencias al vector de Poynting.

La altura del pico de la figura 4 está en $2.3124 \times 10^{-11}$ Watts $/ \mathrm{m}^{2}$. Es una curva de dispersión con un máximo en $\mathrm{l}=378.77 \mathrm{~nm}$ y disminuye hasta casi el $10 \%$ de su valor (lado izquierdo de la curva) en $\mathrm{I}=442 \mathrm{~nm}$. A su vez, a la derecha del pico decrece a la misma intensidad en $346 \mathrm{~nm}$. La nanopartícula selecciona las frecuencias de esta manera.

La curva de dispersión se encuentra en la región de la radiación ultravioleta cercana y solamente el lado izquierdo de la misma se ubica en el rango del color azul. Es lo que llaman una manifestación de la presencia del plasmón.

Este resultado nos permite estudiar la imagen de la figura 5a. La actividad que se encuentra en ésta se debe a la penetración de la luz ultravioleta y azul en los rangos de frecuencia mencionados y son las involucradas en la actividad de los electrones en la superficie de la nanopartícula. Debe notarse que las celdas de actividad se encuentran principalmente en la superficie.
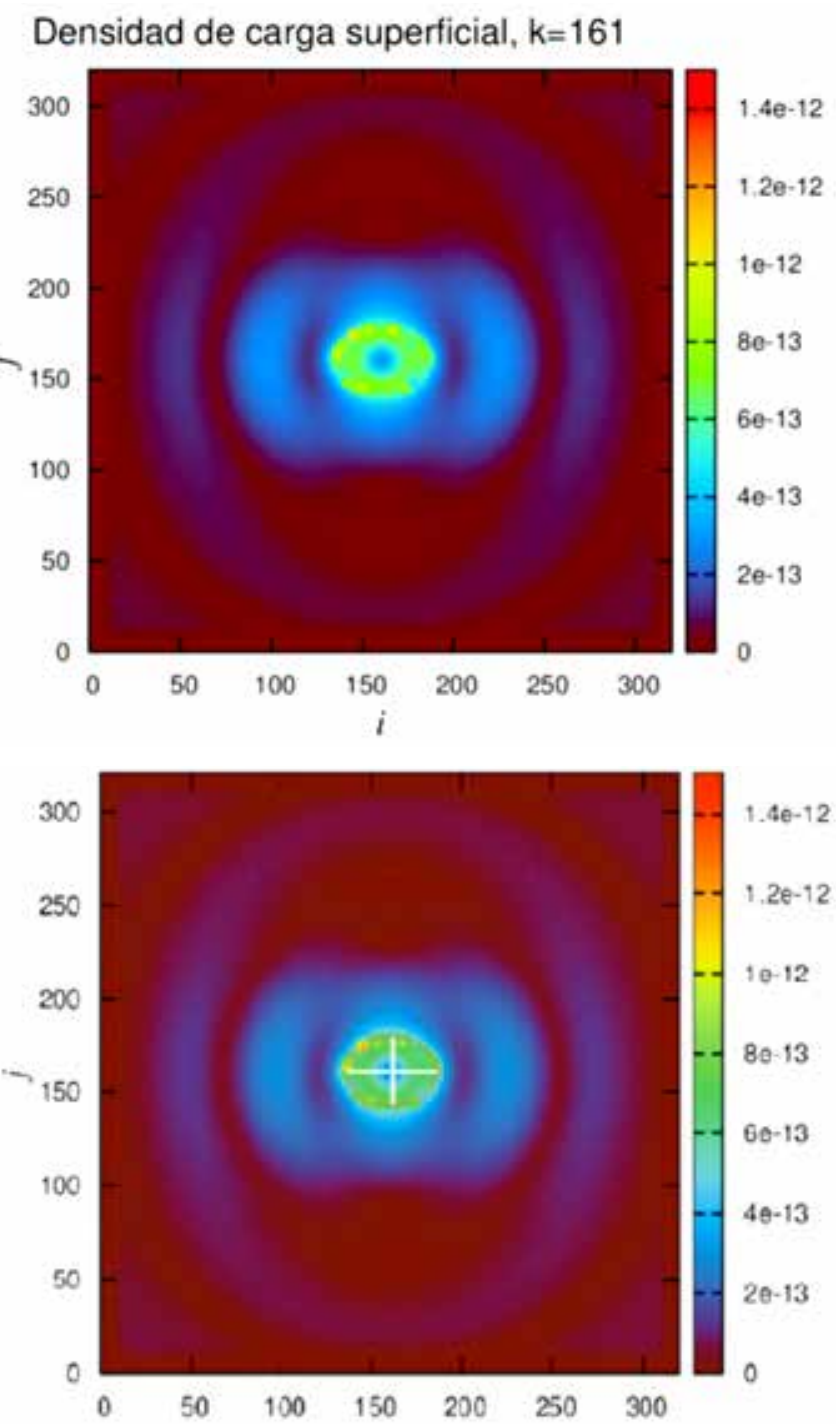

Figura 5a. (Izquierda). Magnitud del campo electromagnético en el plano $k=161, t=1000 \mathrm{TA} ; 5 \mathrm{~b}$ (Derecha). Muestra la figura 5 a con medidas de la nanopartícula agregada. 
Cuando el pulso electromagnético incide sobre una nanopartícula esferoidal prolata, se genera en ésta un movimiento de electrones que forman las corrientes de remolino que han sido analizadas por Tomchuk y Grigorchuk [15], quienes lo atribuyen a un mecanismo en el que interviene la ley de Faraday. Consiste en que el campo magnético de la onda electromagnética genera en torno suyo un campo eléctrico. A su vez este último arrastra corrientes locales de electrones, como se puede ver en las figuras $5 \mathrm{a}$ y $5 \mathrm{~b}$. En ésta se encuentran celdas de actividad que asociamos con el estudio teórico mencionado.

Las medidas de la nanopartícula de plata son: un eje mayor de 50 $\mathrm{nm}$ y un eje menor $35.7 \mathrm{~nm}$. La escala de la figura obtenida permite extraer un segmento de recta de $50 \mathrm{~nm}$ y establecer proporciones con rectas trazadas sobre la imagen de la nanopartícula.

Considerando lo anterior, modificamos la figura $5 a$ para confeccionar la figura $5 \mathrm{~b}$. En esta última se introducen los siguientes cambios: 1) superponemos una elipse que marca el contorno de la partícula esferoidal prolata; 2 ) se agregan los ejes mayor y menor; y 3) se introduce una línea inclinada desde la superficie de la nanopartícula hasta la región donde pierde actividad el campo electromagnético.

De este modo es posible obtener varias dimensiones: el tamaño aproximado de la región central que registra menor actividad (color azul más intenso) y la extensión de la celda de actividad que se aprecia arriba a la izquierda (color amarillo y naranja). Los datos encontrados son los siguientes: a) la celda de mayor actividad (arriba a la izquierda) es de aproximadamente $4 \mathrm{~nm}$; b) la penetración en la región de la celda de mayor actividad es de $9.5 \mathrm{~nm}$; y c) el centro de menor actividad, en el centro de la nanopartícula, mide aproximadamente $19 \mathrm{~nm}$.

La figura 5 a permite apreciar que la actividad ocurre esencialmente en la superficie, más una región exterior en donde el campo electromagnético es muy intenso. Interpretamos que se trata del fenómeno de plasmones de superficie; entendido éste en el sentido de que son regiones donde se presenta el acoplamiento del campo con la vibración de los electrones del material. Existe además una onda evanescente que de acuerdo a la descripción clásica sigue un decaimiento exponencial de su amplitud. Es la respuesta típica de los metales a los campos electromagnéticos incidentes. Éstos penetran una distancia media $\delta$ que está dada por la siguiente expresión [12]:

$$
\delta=\sqrt{\frac{2 \rho}{\omega \mu}}
$$

Se le llama efecto pelicular (skin depth) y consiste en que si Eo es la amplitud de la onda, se reduce aproximadamente al $36.79 \%$ de ese valor en cuanto penetra la distancia media $\delta$. En la expresión anterior, $r$ es la resistividad del material, $c$ es la velocidad de la luz en el vacío, $\mathrm{m}$ es la permitividad del material y $\mathrm{w}$ es la frecuencia de la onda electromagnética. Si denotamos como I a la longitud de onda de la radiación y tomamos en cuenta que ésta es inversamente proporcional a $\omega$, de la ecuación (9) resulta que la penetración $\delta$ crece proporcional a la raíz cuadrada de I. Por ejemplo, para el caso de la resistividad de la plata, la región de la radiación ultravioleta cercana, y la región del color azul, se obtiene que toma valores entre 2.35 y $2.40 \mathrm{~nm}$. Esto se debe a que la luz ultravioleta y azul excitan los electrones de la banda de conducción de la plata de la nanopartícula. Este resultado nos da la explicación del $75 \%$ de toda la celda de mayor actividad. El $25 \%$ restante se debe al movimiento difusivo de los electrones de conducción [16] y [17].

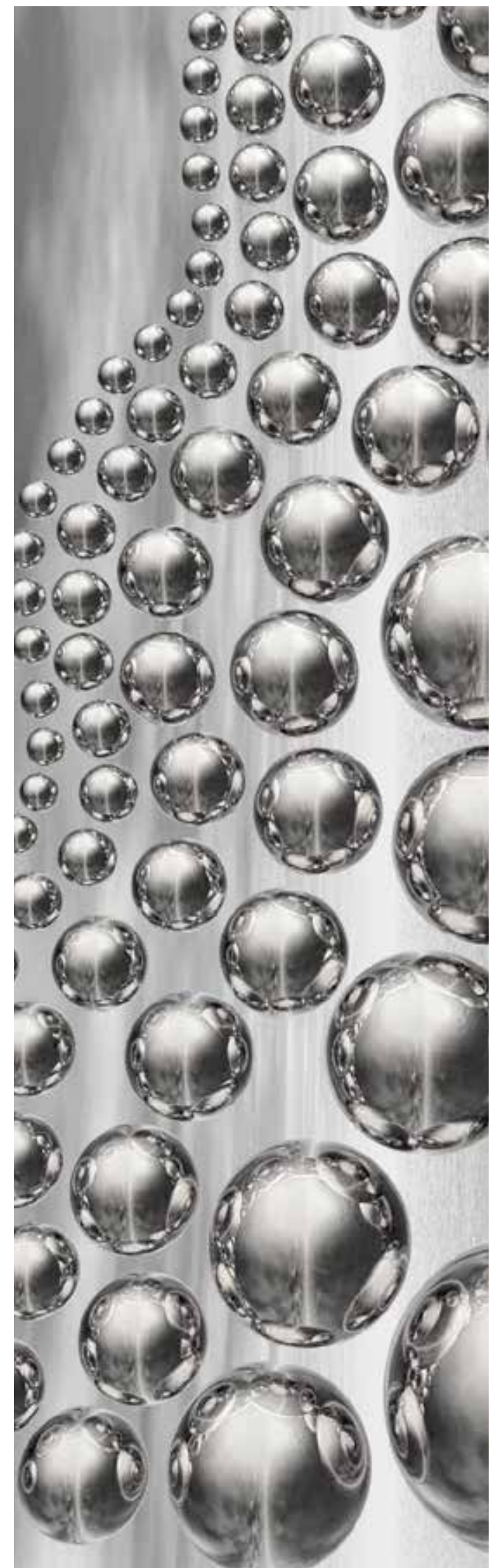




\section{CONCLUSIONES}

Se ha expuesto el método de diferencias finitas en el dominio del tiempo para estudiar la dispersión de luz por una nanopartícula de plata. Se incluye una explicación de la forma del pulso electromagnético utilizado para la solución numérica de las ecuaciones de Maxwell. Se postula que el material responde conforme a lo descrito por el modelo de Drude para los electrones de conducción, de modo que hemos agregado una descripción breve de la ecuación involucrada. La solución numérica permite calcular el vector de Poynting cuando la radiación de la partícula se encuentra en un estado casi estacionario. La transformada de Fourier del mismo presenta un pico muy agudo con un máximo en $\mathrm{I}=378.77 \mathrm{~nm}$, situado en la región ultravioleta. Se obtiene un mapa del estado de radiación justo momentos después de que el pulso perturbador ha dejado de actuar sobre la nanopartícula, con lo cual se identifican las zonas de mayor actividad radiactiva de la nanoestructura. Se encuentra una región de gran actividad, que ha sido medida a escala para conocer sus dimensiones geométricas y el grado de penetración de la radiación en el metal. Los resultados son satisfactoriamente comparados con el efecto pelicular (skin depth) predicho por la teoría y con el fenómeno de difusión de electrones mediante datos experimentales de su trayectoria libre media en la plata.

\section{AGRADECIMIENTOS}

Agradecemos la hospitalidad del Área de Cómputo de Alto Rendimiento de la Universidad de Sonora (ACARUS), donde fue realizado el trabajo numérico.

\section{BIBLIOGRAFÍA}

[1] M. Ranjan Gartia et al., "Colorimetric Plasmon Resonance Imaging Using Nano Lycurgus Cup Arrays," Adv. Optical Mater., Vol. 31, pp. 68-76, Jan 2013.

[2] Consultar, por ejemplo: J. H. Grossman and Scott E. McNeil, "Nanotechnology in Cancer Medicine", Phys. Tod., Vol. 65, no. 8, pp. 38-42, Aug. 2012. J. Dawson, "NSF centers to study societal impact of nanotechnology", Phys. Tod., Vol. 59, no. 2, p. 20, Feb 2006. C. C. M. Mody, "The larger world of nano", Physics Today, Vol. 61, no. 10, pp. 38-44, Oct 2008.

[3] R. Alam R. et al., "Probing Bioluminescence Resonance Energy Transfer in Quantum Rod-Luciferase Nanoconjugates", ACS Nano, Vol. 10, pp. 1969-1977, Oct 2016.

[4] A. O. El-Ballouli, E. Alarousu, A. R. Kirmani, A. Amassian, O. M. Bakr, O. F. Mohammed, "Overcoming the Cut-Off Charge Transfer Bandgaps at the PbS Quantum Dot Interface", Adv. Funct. Mater, Vol. 25, pp. 7435-7441, Dec 2015.

[5] P. M.Tomchuk y N. I. Grigorchuk, "Shape and size effects on the energy absorption by small metallic particles". Phys. Rev. B, Vol. 73, no. 15, pp. 155423-1 - 155423-17, Apr 2006.

[6] F. Delogu, "Thermodynamics on the nanoscale", J. Phys. Chem. B, Vol. 109, pp. 21938-21941, Sep 2005. C. C. Yang, Y. May, "Thermodynamics at the nanoscale: A new approach to the investigation of unique physicochemical properties of nanomaterials", Mater. Sci. Eng. R-Rep., Vol. 79, pp. 1-40, May 2014.

[7] D. Cela et al., "Resource Letter N-1: Nanotechnology", Am. J. Phys., Vol. 82, pp. 8-22, Jan 2014.

[8] A. G. Smart, "Phonon spectrometry goes nanoscale", Phys.
Tod., Vol. 67, pp. 16-17, Feb 2014.

[9] D. Maclsaac, "American Chemical Society ByteSize science wideos, bytesizescience.com/index.cfm", The Phys. Teach., Vol. 51, No. 2, p. 125, Feb 2013.

[10] A. Junka y F. Riess, "From an idea to a vision: There's plenty of room at the bottom", Am. J. Phys., Vol. 74, pp. 825-830, Sep 2006.

[11] K. S. Yee, "Numerical solution of initial boundary value problems involving maxwell's equations in isotropic media". IEEE Transactions on Antennas, Vol. 14(3), pp. 302-307, MayJun 1966.

[12] R. P. Feynman, R. B. Leighton, M. Sands, "The Feynman Lectures on Physics", Addison-Wesley, 1964.

[13] Una presentación didáctica se encuentra en: A. Salvia, "Métodos numéricos para la resolución de ecuaciones diferenciales. Notas del Curso: Cálculo Numérico (plan 87), Universidad de la República - Uruguay, Facultad de Ingeniería", 2002. En línea en: http://www.fing.edu.uy/inco/ cursos/numerico/ed/final/FDTD.pdf.

[14] L. de la Peña, "Introducción a la Mecánica Cuántica", UNAMFCE. 2006.

[15] D. R. Penn, "Electron mean-free-path calculations using a model dielectric function", Phys. Rev. 35, No. 2, p. 482-486, 1987.

[16] H. Kanter, "Slow-Electron Mean Free Paths in Aluminum, Silver, and Gold", Phys. Rev. B, Vol. 1, no. 2, pp. 522-537, 1970.

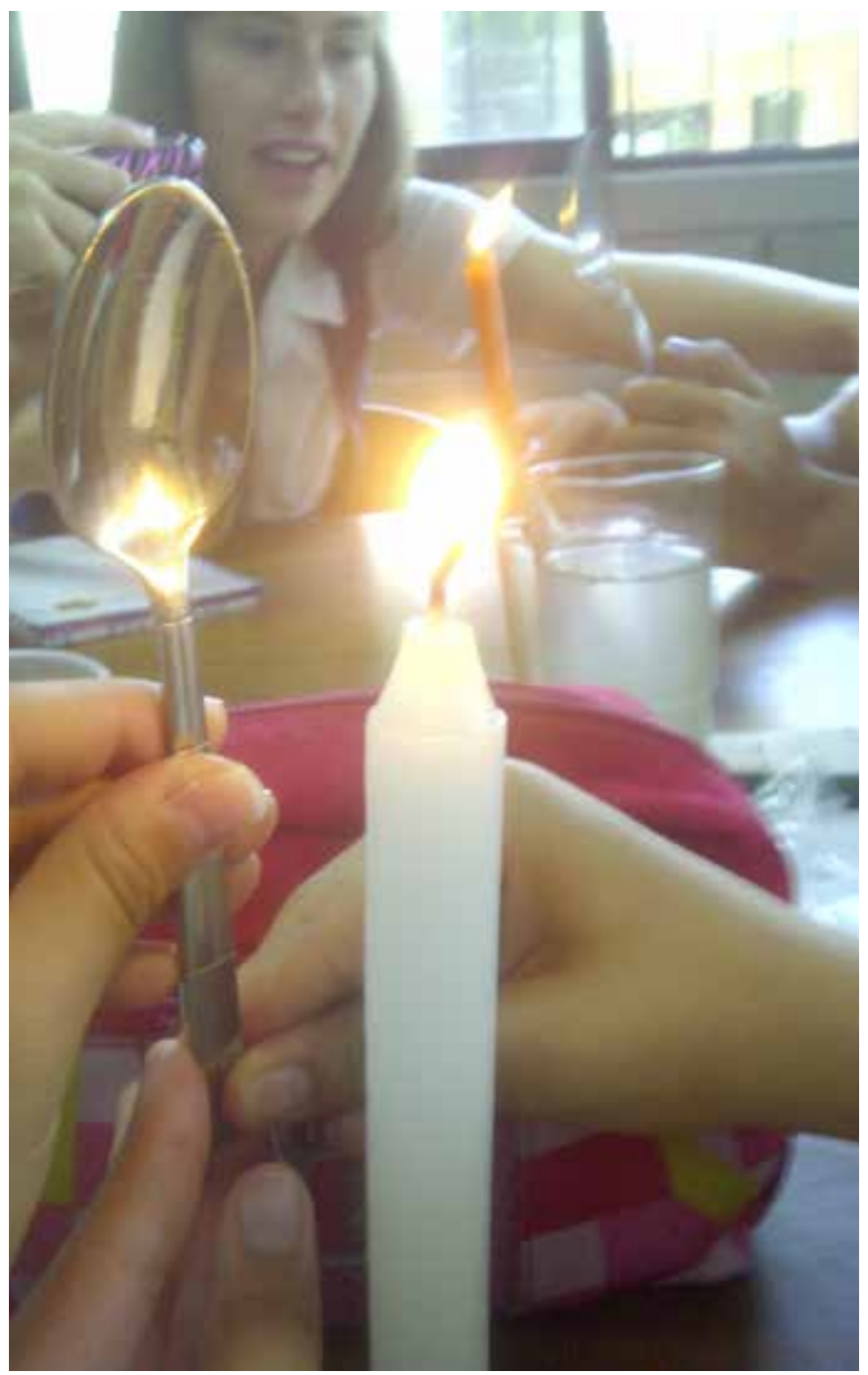

